\title{
MODELOS EPISTÉMICOS, INVESTIGACIÓN Y MÉTODO
}
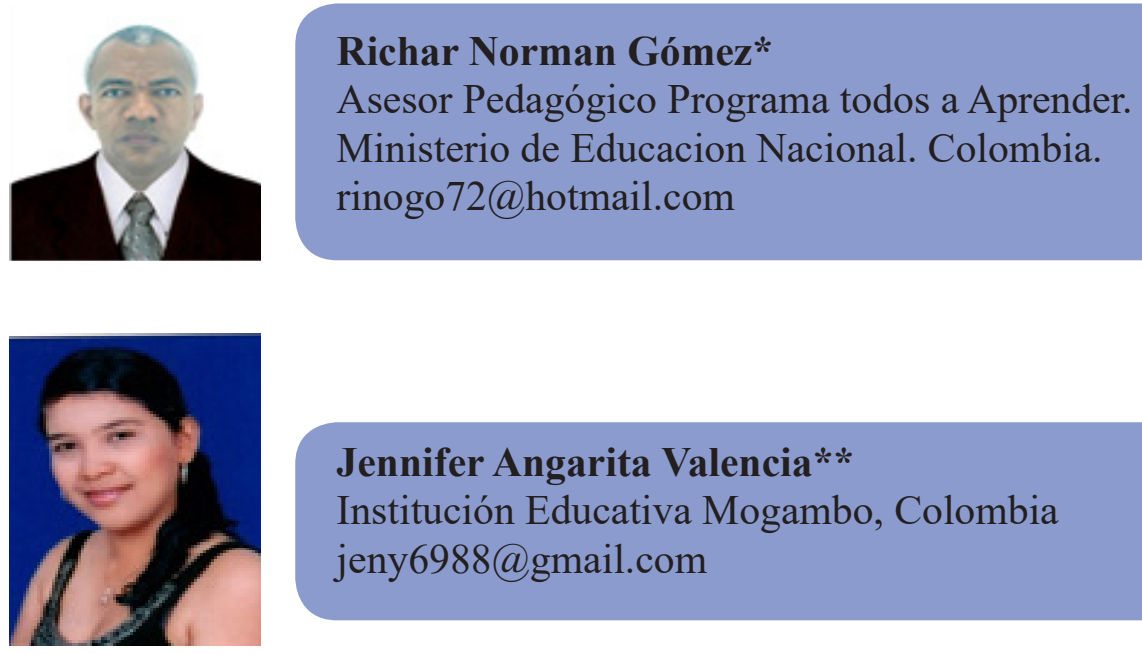

\section{Jennifer Angarita Valencia**}

Institución Educativa Mogambo, Colombia jeny6988@gmail.com

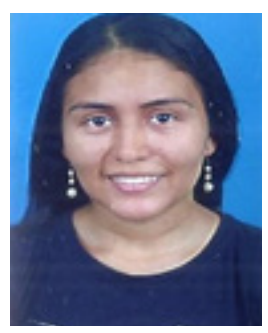

\section{Claudia Beatriz Díaz González***}

Institución Educativa Cristobal Colon, Colombia

cladiagon@gmail.com

DOI: $10.37594 /$ oratores.n13.416

Fecha de aceptación:03/09/2020

\section{RESUMEN}

El objetivo de este documento es presentar una aproximación al concepto de investigación, definida fundamentalmente como un "conjunto de procesos sistemáticos, críticos y empíricos que se aplican al estudio de un fenómeno o problema, con el resultado u objetivo de ampliar su conocimiento" (Hernández \& Mendoza, 2018), es decir que partiendo de una problemática podemos encontrar una solución a ella, bajo un estudio aproximado al fenómeno, desde diferentes modelos epistémicos y hacer un recorrido por los métodos de investigación de cada modelo, como elementos integrales de la vida del ser humano y que lo lleva a cuestionarse constantemente. Con esto se busca una explicación o solución a los fenómenos y problemas que suceden cotidianamente, intentando mejorar el entorno circundante, el estilo, ritmo y calidad de vida del ser humano a partir de horizontes conceptuales claros, que contribuyan de forma holística e integral en la construcción

*Licenciado en Matemáticas y Física, Universidad de Córdoba, Colombia, docente Tutor del Programa todos a Aprender Del Ministerio de Educación Nacional. Docente catedrático de la Universidad Cooperativa de Colombia. **Docente Catedrático Corporación Universitaria Remington.*** Docente Catedrático de la Universidad Santo Tomás. Magíster en Educación, Universidad de Córdoba- SUE Caribe. 
de un mejor y próspero futuro. Logrando así, ampliar las fronteras del conocimiento con procesos metódicos y análiticos cada vez más articulados y razonables, con los objetivos aplicados a diversos estudios de múltiples eventos en los variados campos del saber, lo cual se retoma desde la postura de varios autores dando validez y consistencia intelectual a los planteamientos descritos.

Palabras clave: Investigación, Modelos, métodos, epistémicos.

\section{EPISTEMIC MODELS, RESEARCH AND METHOD ABSTRACT}

The objective of this document is to present an approach to the concept of research, defined fundamentally as a "set of systematic, critical and empirical processes that are applied to the study of a phenomenon or problem, with the result or objective of expanding their knowledge" (Hernández \& Mendoza, 2018), meaning that starting from a problem we can find a solution to it under an approximate study of the phenomenon, from different epistemic models and make a tour of the research methods of each model, as integral elements of life of the human being and that leads him to constantly question himself. This seeks an explanation or solution to the phenomena and problems that occur daily, trying to improve the surrounding environment, style, rhythms and quality of life of the human being from clear conceptual horizons, which contribute in a holistic and integral way in the building a better and prosperous future. Achieving this, expanding the frontiers of knowledge with methodical and analytical processes increasingly articulated and reasonable with the objectives applied to various studies of multiple events in the various fields of knowledge, which is taken from the position of several authors giving validity and consistency intellectual to the described approaches.

Key words: Research, models, methods, epistemic.

\section{INTRODUCCIÓN}

Desde el inicio de la humanidad se ha considerado la adquisición del conocimiento como fuente de toda verdad y de acercamiento a la realidad subyacente. A ello, no escapa la investigación como actividad permanente del hombre, dado que, por medio de ella se busca responder las inquietudes y preguntas a diversos problemas. Es así, como se generan los distintos modelos que conocemos actualmente y que serán guías de trabajo para los investigadores. "La investigación es una actividad basada en el trabajo de otros investigadores, y que puede repetirse" (Salkind, 1998); porque a partir de esas investigaciones ya realizadas pueden surgir otras que ayuden a ver el futuro 
de una manera diferente. Un modelo no es algo acabado, sólo es una representación de la realidad a la que se intenta resolver, por lo cual, es correcto afirmar, que no hay un modelo finalizado, perfeccionado ni culminado, dada su naturaleza evolutiva y progresiva. En este sentido, "no existe un modelo exacto, ni exclusivo, ni puro" (Barrera, 2010a); en investigación se considera correcto el uso de cualquier modelo siempre y cuando su proceso, método o ruta lleve al investigador a resultados que aporten a la situación conjeturada.

\section{¿Qué es investigación?}

Ahora bien, ¿qué entendemos por investigación?, diferentes autores se han realizado esta pregunta y de alguna manera han influenciado o se convirtieron en la base para otros investigadores, permitiendo crear un fenómeno cíclico que a través del tiempo ha avanzado en teorías y nuevos conocimientos.

Un ejemplo trascendental del uso de la investigación desde tiempos antiguos lo hace Cohelet, rey de Israel, al decir: "me he dedicado a investigar y observar con sabiduría todo lo que se hace bajo el cielo" Eclesiastés (1:13). Entendiéndose que el proceso de investigación proporciona nuevos conocimientos gracias a la indagación, reflexión y razonamiento continuo del mundo circundante.

Por su parte, el Doctor Ángel Díaz Barriga ${ }^{1}$ durante un seminario de currículo e investigación afirmó que, "la investigación es la curiosidad innata del ser humano", dando como ejemplo la insistencia y pasión de los niños en las primeras etapas de la vida, alrededor de los 5 años, de preguntar el porqué de todo lo que les rodea, haciendo referencia que la investigación no es competencia exclusiva de expertos y que es la capacidad natural que tiene el ser humano para resolver problemas e indagar acerca del funcionamiento del mundo que lo rodea, la cual se puede afinar con el tiempo, a partir del análisis de la realidad apoyada en evidencias y argumentaciones de rigor, usando horizontes conceptuales, pertinentes y claros para sustentar los procesos ejecutados e inferir las explicaciones, aplicaciones y utilidad de los posibles hallazgos realizados o por el contrario dejarla rezagada y disminuir o perder esta cualidad o aptitud al pasar de los años.

En este mismo orden, teniendo como eje fundamental la investigación, se presentan las siguientes conceptualizaciones de diversos autores con el fin de enfatizar la importancia de este proceso en la vida cotidiana del ser humano:

1 Ángel Díaz Barriga,Doctor en Pedagogía por la Facultad de Filosofía y Letras de la UNAM.Miembro de la Academia Mexicana de Ciencias. Invitado de honor a Seminario de currículo e investigación, Universidad de Córdoba, (2015). Montería, Córdoba, Colombia. 
Sierra (1991) "considera que genéricamente, la investigación es una actividad del hombre orientada a descubrir algo desconocido." (Arias, 2012). Donde referencia la investigación como una capacidad para producir un efecto y que es propia del ser humano, para indagar cosas desconocidas dentro de su contexto académico, social y cultural.

Por su parte, "Sabino (2002) menciona que la investigación puede definirse como un esfuerzo que se emprende para resolver un problema, claro está, un problema de conocimiento." (Arias, 2012). Mostrando la investigación como una acción proactiva necesaria para resolver algo, con un fin determinado y relacionada con la actitud e iniciativa del ser humano en perfeccionar sus ideas y encontrar respuestas a lo desconocido; por esta razón, y de manera innata el ser humano busca las estrategias y los mecanismos para llegar a encontrar la soluciones a sus inquietudes.

Así mismo, "Cervo y Bervian (1989) definen la investigación como una actividad encaminada a la solución de problemas. Su objetivo consiste en hallar respuestas a preguntas mediante el empleo de procesos científicos." (Arias, 2012). Estableciendo la investigación como una capacidad adquirida para dirigir o indicar el camino en la búsqueda de posibles soluciones o respuestas a problemas, a partir del rigor de las ciencias puras o aplicadas, lo que implica descubrir y producir nuevos conocimientos, enmarcados en los aspectos analizados u objetos investigados. Cualquier ser humano puede crear una teória siempre que tenga la disposición de indagar sobre el tema o problema de estudio, en diferentes fuentes confiables que le proporcionen la información necesaria para contrastar la hipótesis ya planteada con los datos investigados, y así transformar la realidad.

Es decir, la investigación posee una característica universal, puesto que "los resultados obtenidos contribuyen a aumentar el patrimonio científico y cultural de la humanidad" (Hurtado, 2012). Ningún estudio desde el primero realizado alcanza a responder todas las inquietudes presentadas, esto se debe a las necesidades y características del contexto histórico, político y social, tampoco el último culminará con todas las dudas sobre el tema en cuestión, dado que todas las investigaciones se aportan, se comparten y se renuevan en un espiral de conocimientos y experiencias adquiridas.

Toda investigación se basa en una teoría debidamente reflexionada, con razonamiento lógico y hechos veraces de situaciones generalizadas dando cuenta de la autenticidad de un proceso. Esta construcción de ideas buscan proporcionar respuestas a interrogantes que generan en todo individuo controversia o negociación de lo que se sabe con lo que se debe saber. Determinar la naturaleza de los hechos que se estudian permite no imponer juicios o hipótesis erróneas sobre la realidad que se investiga y en cierta medida mejorar la sociedad. La necesidad de hacer investigación es inherente 
al carácter reflexivo del hombre porque precisa diversos puntos de vista y formas de entender el mundo; ya sea para encontrar leyes o proposiciones, o simplemente responder a necesidades o inquietudes específicas.

En síntesis, la investigación es la necesidad del ser humano de entender, comprender, asimilar y explicar los diversos fenómenos del mundo que lo rodea, a partir de elementos conceptuales que sustenten las deducciones o inferencias realizadas, permitiendo resolver situaciones con claridad de manera argumentativa y propositiva; siguiendo la ruta que más le convenga al investigador permitiéndole cuestionarse y entrelazar los conocimientos adquiridos en los diferentes ámbitos físico, social, económico, cultural, entre otros.

A continuación, se realiza un acercamiento a la definición de investigación desde los diferentes modelos epistémicos y sus métodos, permitiendo una visión más amplia de estos tópicos intelectuales como evidencia de la autenticidad y particularidad de cada uno como modelo.

\section{Modelos epistémicos}

Todo modelo tiene su manera de ver la realidad, un paradigma que determina sus principios y leyes. La manera como un modelo aprecia el mundo circundante constituye una herramienta fidedigna, verídica y auténtica para generar nuevas experiencias. En términos de Barrera (2010) "un modelo permite entender lo que se percibe y, en consecuencia, propiciar el actuar" (p. 11) dado su característica cambiante, variacional y modificable en términos de produción de ideas y evidencias claras del objeto en cuestión. "Los modelos evolucionan: surgen, sí, pero luego toman diversos caminos" (p.12). Es decir, un modelo constituye una representación abstracta o factual del contexto que se estudia o se intenta explicar, siendo ejemplo o referencia para otros posibles modelos que surgen a partir del mismo; un modelo es susceptible a cambios y transformaciones que pueden llevarlo a su evolución o estancamiento, pero debidamente respaldado por una fundamentación teórica que acredite y avale su existencia y utilización.

Un modelo es emergente cuando surge o se deriva de una confrontación de ideas que desajustan los patrones o constructos establecidos originalmente, buscando como fin último ahondar en situaciones adversas y de escaso bagaje conceptual que de acuerdo al contexto precisan una capacidad representacional mas razonable y rigurosa, conduciendo así al conocimiento y comprensión de diversas situaciones.

Por su lado, un modelo epistémico es entendido como la concepción de un hecho o evento, que como fin último le da pautas al investigador para examinar a profundidad el entorno o circunstancia 
significativa. Para validar la originalidad de un modelo epistémico se hace necesario determinar sus ideas, cosmovisión, lenguaje único y términos propios, valores fundamentales, contextos asociados a su realidad y semiología representativa.Todo modelo epistémico al ser inacabado es susceptible de nuevas ideas emergentes que no lo cambian, todo lo contrario, lo enriquecen o en su defecto se bifurcan e identifican como nuevas representaciones de la realidad. Los modelos epistémicos se clasifican en dos grandes grupos: originarios y derivados.

\section{Modelos originarios}

Son aquellos prototipos que generan reflexión y comprensión en diferentes ramas del conocimiento generando un desarrollo. Los más representativos modelos originarios son: el naturalismo, idealismo, materialismo, realismo, antropocentrismo y ontologismo.

El modelo del naturalismo considera que "la naturaleza es la fuente del conocimiento y orientadora de la praxis humana y social" (Barrera, 2010a); es decir que de las observaciones que se realicen del ambiente, se logra recuperar toda la información pertinente de lo que ocurre, teniendo claridad de lo sucedido; debido a unas leyes y principios establecidos universalmente por la comunidad científica en general. Este modelo se cimienta en todo aquello que se pueda observar teniendo en cuenta los sentidos, donde el conocimiento se fundamenta a todo lo que la naturaleza puede brindar al ser humano. El naturalismo busca en su devenir investigativo obtener sabiduría, mediante el encuentro con la naturaleza o todo fenómeno natural. El método realizado para llegar a este fin es el comparativo, haciendo prelación de lo instintivo, de la inserción, abstracción, observación y definición de contextos.

Por otra parte, el idealismo propone que las ideas son el principio de las cosas y que todo surge a partir de ellas. "La realidad se evidencia de las ideas; las cosas son imperfecciones de las ideas; además, la indagación se concentra en describir dichas ideas, en comprobar lo que se quiere a partir de las ideas previas" (Barrera, 2010a). Es importante que toda investigación parta de una idea de la cual se va a trabajar para afirmar o negar algo; todo gracias a los métodos que se pueden utilizar, en este caso por medio de la contemplación, la generación del conocimiento y la comprobación de lo expuesto anteriormente por otros expertos que en cierta medida aportan a la construcción de la colectividad humana.

En cambio, el antropocentrismo se centra en el hombre, su ser como tal, privilegiando su estudio desde su integridad. Su método tiene como propósito la integración y eulerización comprensiva de la naturaleza y existencia del hombre para estructurar su pasado y evolución en los distintos aspectos que lo caracterizan como eje fundamental en la construcción de conocimiento. 
Por su parte, el materialismo propone el desarrollo de iniciativas relacionadas a procesos físicos y energéticos. Investigar en el materialismo es explicar con base en la lógica, raciocinio y demostración, es decir, en la dialéctica del contexto; investigar en este modelo, es estudiar la sociedad en constante cambio y analizar el origen y desarrollo de los hechos. Su método, el crítico dialéctico se justifica en la particularidad de la inserción en la práctica y en su mayor efecto: el cambio material, Marx citado por Hurtado (2012) afirma que este método "abarca estadios descriptivos, analíticos y explicativos del proceso de investigación", donde se describen de manera detallada las situaciones, realizando un análisis sobre los aspectos relevantes que se descubren al momento de hacer la investigación, como elementos fundamentales en su conceptualización.

Por el contrario, el realismo tiene como propósito generar saberes a partir de la realidad, la validez del conocimiento es determinante. La confirmación de los hechos depende de la observación. "El realismo privilegia el estudio y comprensión del ser humano ante el universo para determinar la manera de apreciarlas y en consecuencia de actuar" (Barrera, 2002). Por lo tanto, la realidad se estipula de acuerdo a los conocimientos y vivencias experienciales; asimismo, el realismo sostiene que la forma como se desarrollan las cosas y las maneras de participar de ellas, es por medio de la experiencia, la cual constituye la única y real fuente de saber.

Por último, el modelo del ontologismo, muy similar al antropocentrismo, busca reconocer la existencia del ser y el ser en cuanto que existe y es pensado. Esta aprehensión del ser es a través de la contemplación, de la praxis, de la intuición, de la construcción o de la investigación en sí, tratando aspectos desde su incidencia holística e integral.

\section{Modelos derivados}

Los modelos derivados son esas vertientes o ramificaciones que se producen de los modelos originarios, dándole más fortaleza a las maneras específicas de hacer investigación. Son varios los modelos derivados existentes según la forma de proceder para alcanzar los logros propuestos y así comprender los pasos que guían la acción; existe la posibilidad del surgimiento de nuevos modelos derivados a partir de los conocidos científicamente y de acuerdo al contexto, dependiendo principalmente de los fenómenos estudiados y los problemas declarados en cada uno de ellos.

Dentro de ellos se encuentra el pragmatismo, derivado del empirismo; en el cual se valora el conocimiento a partir de la actividad práctica para conocer y sustentar lo conocido con la capacidad explicativa racional. Es decir, investigar un problema es resolverlo desde la práctica, en otras palabras, el valor del conocimiento está en la práctica. El pragmatismo, busca brindar las 
soluciones necesarias y factibles, al momento mismo de la investigación, utilizando los materiales que se encuentren al alcance. Este modelo surge a partir de las necesidades del individuo y la sociedad para obtener soluciones prácticas y rápidas.

El método aplicado en este modelo es la investigación - acción, porque implica por parte del investigador "una acción planificada y dirigida al logro de ciertos objetivos" (Hurtado, 2012). Involucrarse en el proceso investigativo es parte fundamental en este método, dado que todo investigador determina el grado de incidencia o posible solución a la problemática planteada y en este caso, es necesario comprobar para teorizar y dar a conocer nuevas perspectivas que no trabajas desde la mirada de un observador no participante. Este método, intenta identificar los cambios efectuados por la actividad práctica y modificarlos, dado que "la acción proporciona la base de la racionalidad concreta, única que posee el hombre” (Hurtado, 2012). Razón por la cual, la acción práctica se convierte en el génesis de todo principio científico que busca ser aprobado pero que desde luego fue estudiado a profundidad.

En cambio, en el positivismo la investigación es considerada como "la verificación de hipótesis en las cuales se plantean relaciones causales entre diferentes fenómenos" (Hurtado, 2012). En este modelo se privilegian los hechos ante las ideas, a las ciencias experimentales ante las teorías y leyes físicas. Todo debe ser comprobado para obtener el nivel de lógico, racional y verificable de autenticidad científica. Al ser un modelo derivado del realismo, su método hipotético - deductivo busca obtener nuevos conocimientos a través de la observación, la experimentación y formulación de hipótesis; se plantea "en el positivismo que la ciencia - con criterios de verificación - es el objetivo de todo conocimiento; el conocimiento debe estar relacionado con el desarrollo de la historia y de la sociedad para que sean realmente válidos" (Barrera, 2010a). Ninguna teoría por muy innovadora y revolucionaría que sea debe ser validada para ser creible, de lo contrario se convierte en seudociencia, sólo interpretada, sin ser comprobada. Así mismo, "en este método se contrastan las hipótesis derivadas de una teoría, pero no para construir teorías" (Hurtado, 2012), sólo busca verificar si las hipótesis planteadas con respecto al problema se cumplen teniendo en cuenta la teoría descrita en el marco teórico. Eso no significa que se elaborarán nuevas teorías a partir de lo encontrado en la investigación; sólo se anexa información no valorada por la utilización de paradigmas que desdibujan el surgimiento de nuevos conocimientos.

Por el contrario, investigar en el estructuralismo es identificar la estructura que da cuenta del objeto de estudio. En éste modelo, investigar es interpretar y explicar a partir de las relaciones que se establecen en el proceso. Además, éste modelo busca aclarar o resolver los vinculos que existen entre el comportamiento del ser humano, en lo social y personal. Su método de análisis 
estructural según Levi-Strauss (1974), citado por Hurtado J. (2012) manifiesta que se debe avanzar en 3 etapas: “observación de lo real, construcción de los modelos y analizar la estructura”. Donde la observación es la base para el inicio de la investigación, luego se construye un modelo teórico para comprender con exactitud lo que se está observando, para así realizar un análisis que permita identificar los elementos del problema y como guardan relación entre sí. Otros autores, definen a este método como etnográfico, donde se tiene en cuenta el contexto del fenómeno e interpretación de un conjunto de relaciones analógicas, coherentes y articuladas entre si con el fin de visualizar la estructura del objeto estudiado.

Por su parte, el empirismo procedente del realismo, está basado en la vivencia y la experiencia, cuyo propósito según plantea Barrera (2010b), es aceptar la forma como se desarrollan las cosas y las maneras de participar en ellas, a partir de la experiencia, la que constituye la única y real fuente de saber. El empirismo considera la investigación como proceso que accede a reflejar la realidad de manera fiel y con neutralidad, permitiendo la expresión individual como forma de mostrar la conciencia colectiva que se percibe del mundo. "Investigar es básicamente crear conocimiento a través de la descripción de la realidad" (Hurtado, 2012). La base de todo el proceso de indagación radica en la información obtenida a partir del contexto donde está inmerso el objeto de investigación del fenómeno analizado.

En este mismo sentido, la fenomenología modelo derivado del empirismo, tiene como propósito saber lo que sucede con base en el evento de estudio, tratando de extraer las particularidades y descubriendo sus características; todo esto se logra con la observación, concentración y percepción. Para este modelo "el investigador se coloca en actitud de explorar simplemente lo dado y para ello deja de lado las creencias y los juicios" (Hurtado, 2012). Generando así nuevas perpesctivas frente al conocimiento, aludiendo a su criterio y espíritu investigador. Este modelo busca describir las experiencias del investigador tal y como se muestran al conocimiento, sin ninguna duda o condición que lo aten a uno u otro paradigma. El método de la fenomenología no supone las cosas y sólo desea explorar lo que el evento está brindando en plenitud, partiendo de cada una de las vivencias.

Por esta razón Hernández (2000) citado por Hurtado plantea 3 pasos que se deben tener en cuenta al realizar una investigación, ellas son: descripción, reducción y búsqueda de esencias; donde el evento se describe tal y como se observa, el investigador apoya el evento con una vivencia original y distinta pero relacionada; para luego conectarlo con el evento de estudio. Básicamente, la fenomenología se enfoca en la naturaleza de la experiencia compartida por cada individuo ante un hecho en común, dando a conocer sus sentimientos, emociones, razonamientos y percepciones directamente relacionados en un contexto en particular. 
En el modelo del cognitivismo, "el conocimiento es visto como proceso, aprehensible desde la condición cibernética y técnica, con respecto a las partes y las funciones de una unidad informática" (Barrera M. , 2010b); donde estudia teóricamente los procesos mentales con el fin de entender la conducta humana estableciendo el comportamiento o aspectos cognitivos, que ayudan en el procesamiento de la información y se evalua la forma como se adquiere el conocimiento, a través de unas habilidades y competencias; realizando un procesamiento de la información a través de diferentes representaciones mentales. Este modelo, se conoce también como cibernetismo, donde "el conocimiento tiene validez en la medida que es comparado, guiado y asociado con la interacción propia del procesamiento, incluye la interacción con redes, la publicación instrumental en bases de datos, con internet y formas lnfo" (Barrera M. , 2010b); es decir, la actividad intelectual es dinámica, agil, rápida y principalmente transitoria, donde se detecta que el ser humano constantemente transita en procesos que no son registrados por la conciencia, logrando una trasformación en el ingreso y salida de la información.

El conductismo estudia la vida del ser humano mediante recursos y técnicas instrumentales; teniendo en cuenta lo observado de la conducta, sus características y expresiones permitiendo mantener un control del ser humano. "Para el conductismo, la observación de la conducta es la clave para comprender y así orientar el aprendizaje y la socialización” (Barrera M. , 2010b) ; es decir que la conducta puede ser medida teniendo en cuenta unos instrumentos ya seleccionados, que le permitan desarrollar sus capacidades por medio de estimulos y condiciones ambientales; donde el conocimiento esta asociado con mecanismos de actividad nerviosa. La investigación según el conductismo esta relacionada en causa - efecto; logrando que por medio de estimulos se obtenga una respuesta de la conducta del ser humano. En efecto la conducta del ser humano ha sido estudiada por diferentes autores que promovieron lo que hoy conocemos como test psicológicos permitiendo determinar los rasgos característicos que posee un individuo.

Así mismo, el constructivismo modelo derivado del estructuralismo, según Bunge (1980) "es una creación mental, una producción abstracta, tal como los conceptos, las proposiciones, los contextos y las teorías" (Barrera M. , 2010b), donde los conceptos son las unidades que forman una proposición; la proposición expresa los niveles de verdad de un enunciado; el contexto es el conjunto o grupo que presenta las evidencias y la teoría corresponde a las relaciones lógicas que tienen cada una de estas proposiciones. Todo esto plantea que el conocimiento se presenta como una construcción de la realidad, que se fundamenta de manera organizada, proporcionando las evidencias de los hechos que se organizan de forma mental, tratando de reflejar las relaciones que existen entre las ideas planteadas. 
Finalmente, el racionalismo propio del materialismo, se basa en el pensar, en el abstraer y en los efectos propios del raciocinio, "la investigación es un proceso de razonamiento, donde el conocimiento producido es de carácter generalizante y universal" (Hurtado, 2012,). Esto indica que todos los procesos realizados se basan en la razón a partir de la abstracción como elementos fundamentales en la producción del conocimiento.

\section{CONCLUSIONES}

En el quehacer investigativo se hace relevante que todo investigador conozca y reconozca los diferentes modelos epistemológicos y sus respectivos métodos de acuerdo a las características y procesos de los fenómenos estudiados, permitiéndole un horizonte conceptual definido y claro que contribuya a la toma de decisiones dentro de los diferentes ámbitos de estudio. Además de poder utilizar diferentes métodos y modelos dependiendo de las necesidades que tenga el investigador, ya que es la fuente por la cual puede encontrar la información.

Es clara la concepción y estructura de cada modelo epistémico, sus orígenes y posibles avances en la construcción de nuevos modelos que pueden surgir gracias a los hallazgos obtenidos por los actuales investigadores en las diferentes ramas del conocimiento.

Una investigación requiere, necesariamente de antecedentes que sirvan de base epistemológica, dándole consistencia al proceso investigativo. Los procesos investigativos están presentes en los diferentes campos del saber: sociales, culturales, científicos, políticos, religiosos, educativos, entre otros; y a partir de sus modelos y métodos se pretende dar solución a las múltiples problemáticas a las que el ser humano constantemente se enfrenta en la sociedad, mejorando así el estilo de vida de la humanidad, aportando nuevos hallazgos y conocimientos que permitan ampliar las fronteras del saber.

Es importante resaltar que existen diferentes modelos epistémicos y cada uno propone un concepto de investigación distinto, de acuerdo al contexto donde se generan los fenómenos y las posibles problemáticas, requiriendo del uso de métodos investigativos pertinentes, conforme a las diversas áreas del conocimiento; utilizando en ellos etapas o fases a consideración del investigador como: la indagación, observación, exploración, descripción, comparación y análisis, todas ellas con el propósito de construir un sendero que conduzca a la resolución del problema develado, contribuyendo así en la generación de nuevos conocimientos a partir del objeto de estudio. 


\section{REFERENCIAS BIBLIOGRÁFICAS}

- Arias, F. (2012). El proyecto de investigación. Introducción a la metodología científica. Caracas: Episteme.

- Barrera, M. F. (2002). Modelos Epistémicos . Caracas: Cooperativa Magisterio.

- Barrera, M. F. (2010a). Modelos Epistémicos en investigación y educación. Caracas: Quirón Ediciones.

- Barrera, M. (2010b). Modelos Epistémicos en investigación y educación. Caracas: Quirón Ediciones.

- Hernández, R. S., \& Mendoza, C. P. (2018). Metodología de la investigación. México: Mc Graw Hill.

- Hurtado, J. (2012). El proyecto de investigación. Comprensión holística de la metodología y la investigación. Caracas: Ediciones Quirón.

- Hurtado, J. (2012). Metodología de la investigación. Guía para la comprensión holística de la ciencia. Caracas: Ediciones Quirón.

- La Biblia. (2000). Giner.

- Michel Salazar, J. A. (2006). Sobre el estatuto epistemológico de las ciencias de la educación. Teoría y Didáctica de las Ciencias Sociales. P. 139-157.

- Salas Madriz, F. E. (2003). La administración educativa y su fundamentación. EDUCACIÓN , P. 9-16.

- Salkind, N. (1998). Métodos de investigaciòn. México: Pearson Prentice Hall. 